\title{
Supporting Comedy Writers: Predicting Audience's Response from Sketch Comedy and Crosstalk Scripts
}

\author{
Maolin Li \\ Independent Research ${ }^{*}$ \\ maolin.li.cs@gmail.com
}

\begin{abstract}
Sketch comedy and crosstalk are two popular types of comedy. They can relieve people's stress and thus benefit their mental health, especially when performances and scripts are high-quality. However, writing a script is time-consuming and its quality is difficult to achieve. In order to minimise the time and effort needed for producing an excellent script, we explore ways of predicting the audience's response from the comedy scripts. For this task, we present a corpus of annotated scripts from popular television entertainment programmes in recent years. Annotations include a) text classification labels, indicating which actor's lines made the studio audience laugh; b) information extraction labels, i.e. the text spans that made the audience laughed immediately after the performers said them. The corpus will also be useful for dialogue systems and discourse analysis, since our annotations are based on entire scripts. In addition, we evaluate different baseline algorithms. Experimental results demonstrate that BERT models can achieve the best predictions among all the baseline methods. Furthermore, we conduct an error analysis and investigate predictions across scripts with different styles. ${ }^{1}$
\end{abstract}

\section{Introduction}

Comedy plays a major role in people's lives in that it relieves stress and anxiety (Williams et al., 2005; Sarıtaş et al., 2019). There are two popular types of comedy: sketch comedy and crosstalk. A sketch comedy usually presents a short story and is performed by multiple comedians in various short

${ }^{*}$ The research was conducted during non-working time. The idea of this research was inspired by a discussion with my friend about an entertainment TV programme in which the comedians mentioned the difficulties of producing a highquality script.

${ }^{1}$ The corpus and source code can be freely downloaded from https://github.com/createmomo/ supporting-comedy-writers scenes; while in a crosstalk performance, which is similar to a talk show, there are usually two performers telling humorous stories behind a desk. Although these two types of comedy are different, both of them are performed based on scripts. A script breaks down a story into pieces along with the details that describe which performer should take what action or say which lines at a specific point (Blake, 2014). Therefore, the quality of the script is critical and it directly influences whether the audience enjoys the performance.

However, it is difficult for script writers to ensure a high-quality comedy script and be productive. Firstly, writers have to assess if audiences will react as expected, in particular laughing at specific points. It is necessary to rehearse multiple times to continuously improve the script, which is time-consuming and can be costly. Secondly, to develop laughter triggers, writers need to identify the potential points from the script where there are possibilities for performers to use funny body moves, tone or tell amusing stories to make the audience laugh. Thirdly, the more times a script is publicly performed, the less laughter it can bring, since the audience have become too familiar with it. Thus, it is essential for comedy writers to explore new laughter triggers constantly.

Since natural language processing (NLP) has been widely and successfully applied to a number of fields (Carrera-Ruvalcaba et al., 2019; Rao and McMahan, 2019), we investigate how NLP methods can support comedy writers to produce high-quality scripts more efficiently. This paper specifies this challenge as a new task, i.e. the prediction of the audience's response to sketch comedy and crosstalk scripts. To address this challenge, we explore the use of two different NLP methodologies: 1) Text Classification: we predict whether or 


\begin{tabular}{cll}
\hline Label & Actor's Line & Source \\
\hline 1 & 宋小宝: 我的人生格言是, 在哪里跌倒, 就在哪里睡一觉。 & 碰瓷 (An Incident-Faking Extortionist) \\
& Xiaobao SONG: My life motto is to have a sleep where you've fallen. & Joyful Comedians (Season 1), 2015 \\
\hline 1 & 张小斐: 我可能是洗的你藏私房钱的这条裤子。 & 幸福牛家村 (Happy Niu Families' Village) \\
& Xiaofei ZHANG: The trousers I wash might be the ones you hide your secret purse. & JSTV Chinese New Year Gala, 2019 \\
\hline 0 & 沈腾: 大妈, 你好好回忆一下, 真的没有撞你。 & 扶不扶 (Help Her Up or Not) \\
& Teng SHEN: Please recall exactly what happened. I really did not hit you. & CCTV Chinese New Year Gala, 2014 \\
\hline
\end{tabular}

Table 1: Text classification annotation examples taken from different comedies in our corpus. In the Label column, $\boldsymbol{I}$ and $\boldsymbol{O}$ indicate whether or not this line makes audiences laugh respectively; In the Action Line column, we present the performer's names and their lines; The Source column indicates the title of the comedy and the venue where it is performed.

\begin{tabular}{ll}
\hline Actor's Line with Annotations & Source \\
\hline 贾玲: 这种装修风格显得你家特别的大。(客厅几乎是空的) & 㑛汉相亲 (Idler's Blind Date) \\
Ling JIA: With this decoration style, your house seems to be incredibly big. (The living room is almost empty) & Ace VS Ace (Season 4), 2019 \\
\hline 岳云鸠: 不能, 不退票是我们的服务宗旨。 & 非一般的爱情 (Unusual Love) \\
Yunpeng YUE: No way! Our policy is no refund. & Joyful Comedians (Season 2), 2016 \\
\hline 贾冰: 恩。 (贾冰乘乘地闭上了双眼) & 贾总的演讲 (Manager JA's Presentation) \\
Bing JIA: Okay. (He duly closes his eyes) & Legend of Laughter (Season 1), 2017 \\
\hline
\end{tabular}

Table 2: Information extraction annotation examples taken from different comedies in our corpus. In the first column, we highlight the text spans that trigger laughs from audiences. Note that, we also collected the performer's moves (e.g., "duly closes his eyes" in the third example).

not an actor's lines ${ }^{2}$ can make audiences laugh. In other words, we formulate the task of predicting as a binary text classification problem. 2) Information Extraction: we predict the text spans from an actor's lines indicating the specific words that trigger an audience's laughter.

Contributions Firstly, we introduce a Chinese corpus of annotated comedy scripts collected from popular TV entertainment programmes. Our annotations include both text classification and information extraction labels. Tables 1 and 2 present annotation examples. The corpus can be used to build an intelligent system to benefit the script writing for comedy writers. It may also be useful for dialogue system research and discourse analysis. Secondly, we evaluate a number of NLP methods and the results demonstrate that BERT models (Devlin et al., 2019) are able to achieve the best prediction performance among all methods. We also further conduct an error analysis which may be useful for further improving the performance. Lastly, we experimentally show that our corpus can also be used to predict laughter triggers for scripts which have very different styles compared to training data.

\section{Related Work}

Our work is closely related to humour detection, which has been widely studied for many years in natural language processing. Mihalcea and Strapparava (2006); Yang et al. (2015); Chen

\footnotetext{
${ }^{2}$ The lines are from the dialogue of a comedy performance. Each line consists of an actor's name and the sentences this actor speaks in performance.
}

and Soo (2018); Blinov et al. (2019) investigated if a text fragment is a one-liner. ${ }^{3}$ Zhang and Liu (2014); Ortega-Bueno et al. (2018); Chiruzzo et al. (2019) explored the humour classification task on tweets. Castro et al. (2018) collected humour values and funniness scores of Spanish tweets by using crowdsourcing. Chiruzzo et al. (2019) proposed a regression task that predicts the humour score for a tweet. Li et al. (2020) collected Chinese Internet slang expressions and combined them with a humor detecting method to analyse the sentiment of Weibo ${ }^{4}$ posts. It should be noted that the examples in all of the corpora used or constructed in the above-mentioned studies are independent of each other. Since our corpus is based on entire scripts, the annotated lines and text spans might also benefit the researchers who are interested in modelling long-context-aware algorithms to understand humour. Apart from the studies on short text fragments, Bertero (2019) and Hasan et al. (2019) created corpora from television (TV) sitcoms such as The Big Bang Theory ${ }^{5}$ and TED talks ${ }^{6}$ respectively. Their goal is to predict whether or not a sequence of texts will trigger immediate laughter. Yang et al. (2015); Zhang et al. (2019) extracted the key words such as sing, sign language and pretty handy from jokes, which are similar to our information extraction annotations.

\footnotetext{
${ }^{3} \mathrm{~A}$ one-liner is a joke that is delivered in a single line which only contains a few words.

${ }^{4}$ Weibo is a Chinese micro-blogging website similar to Twitter: https://www. weibo.com/$$
5 \text { https://the-big-bang-theory.com/ }
$$$$
{ }^{6} \text { https : //www.ted.com/talks }
$$ 


\section{Corpus}

\subsection{Data Collection}

Source Selection In order to ensure the highquality of scripts, we carefully selected thirty performances (the total duration is approximately 473 minutes), including both sketch comedies and crosstalks, of which the leading roles are famous Chinese comedians. These performances were played on well-known Chinese TV entertainment programmes such as Chinese New Year Gala and Ace $\mathrm{VS} \mathrm{Ace} \mathrm{C}^{7}$. Since there were many people in the audience present for the recording of these performances, the annotators can judge whether the audience laughed based on the performance videos. Please refer to the appendix for the full list of performances which gives details of their titles, leading comedians and sources. Lastly, we manually typed up actors' lines for each performance and completed thirty scripts. Although there may be differences between our scripts and the real scripts used by comedians in terms of format or content, we assume that our scripts contain the key information about the real scripts, i.e., the actors' lines. Therefore the corpus can be useful for the development of intelligence-assistant comedy script writing systems.

Diversity We also took the comedy style into consideration. In order to ensure the diversity and its balance: a) The performances were selected from three main different types of sources ${ }^{8}$ as shown in Table 3, including the topic descriptions of selected performances. It can be observed that the corpus has a wide range of topics. b) As a preliminary study, we selected six popular Chinese comedians who have various and distinctive styles, and we chose five representative performances of each comedian.

Corpus Statistic Table 4 illustrates the statistics and Figure 1 shows the laughter rates of each script. The highest line-level and character-level rates are

\footnotetext{
${ }^{7}$ https://es.wikipedia.org/wiki/Ace_vs_ Ace

${ }^{8}$ The three sources are: Chinese New Year Galas - the annual televised Chinese New Year celebrations which are the most viewed TV shows in China. The shows consist of various performances including sketch comedies and crosstalks; Reality Shows - the programmes that show the unscripted actions of participants such as playing games and talking. We selected the shows in which comedians were involved; Comedy Competition Shows - the programmes where different comedians present their comedy performances to a studio audience and the winners are selected based on the audience's votes.
}

\begin{tabular}{cl}
\hline Source & Topics \\
\hline Chinese New & - Love stories and blind dates between old people; \\
Year Galas & - Reflecting social phenomena to call for a better society (e.g. avoid \\
& judging people by their appearances, do not spoil children, care \\
& more about lonely seniors, the woman builds a good relationship \\
& with her mother-in-law, spend more time with children, be wary \\
& of scams); \\
& - Funny family stories during spring festival; \\
\hline Reality & - Stories happened in ancient times; \\
Shows & - Stories about young people (e.g. encounter ex-boyfriends or \\
& ex-girlfriends, relationships between best friends, blind dates); \\
& - Reflecting social phenomena to call for a better society (e.g. give \\
& seats to vulnerable people); \\
\hline Comedy & - Love stories; \\
Competition & - Hot topics (e.g. support the COVID-19 frontline fighters); \\
Shows & - Funny stories that happened among friends and in families; \\
& - Reflecting social phenomena to call for a better society (e.g. be \\
& wary of scams, care more about orphans in orphanage); \\
\hline
\end{tabular}

Table 3: Topics of the selected comedies.

\begin{tabular}{ll}
\hline Statistics & Value \\
\hline \# of Comedy Scripts & 30 \\
Year Range & $2014-2020$ \\
Total Duration & 473.44 mins \\
Average Duration & $15.78 \mathrm{mins}$ \\
\# of Actors' Lines & 6087 \\
Laughter Rate (Line-Level) & $28.62 \%$ \\
\# of Characters & 120451 \\
Laughter Rate (Character-Level) & $8.16 \%$ \\
\hline
\end{tabular}

Table 4: Corpus statistics. \# of Actors' Lines and Characters correspond to the total number of lines and characters in our corpus respectively. Laughter Rate is the rate of lines/characters that trigger laughter.

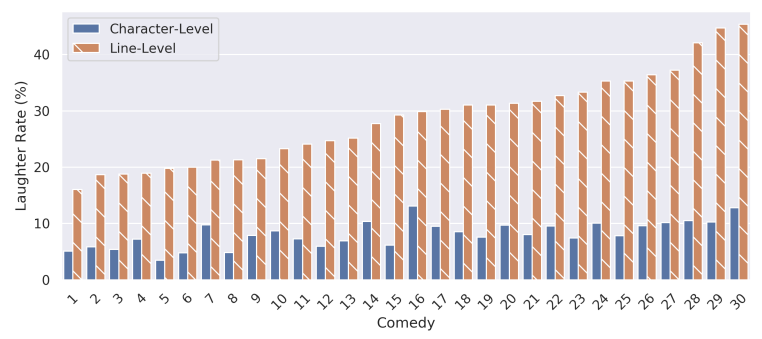

Figure 1: Each script's laughter rate in our corpus.

$45.39 \%$ and $13.12 \%$, while the lowest rates are $16.03 \%$ and $3.49 \%$. We note that the characterlevel laughter rates vary in different scripts. This may be due to density of laughter triggers of a line or the topic of the script.

\subsection{Annotation}

The annotation was completed on Doccano platform (Nakayama et al., 2018) and the annotators are two native Chinese speakers. The annotations were produced based on the studio audiences' responses as observed in the videos, and are not based on the annotators' responses.

Annotation Instruction Annotating text classification labels is easy; annotators are requested to simply assigned label $l$ to the lines that make audiences laugh, and $O$ to the others. With regard to 
the information extraction annotations, annotators are requested to identify text spans which are usually phrases. The span consists of the words that immediately made the audience laugh after the comedians said them. For example, as indicated in Table 2, the span incredibly big was annotated. In this case, only annotating big would be considered as an incorrect annotation, because the comedian was using incredibly to strongly emphasise big which was her first impression of a man's house in a blind date. Only annotating incredibly would also be incorrect, because the main reason why the audience laughed was because the comedian said the house looked big. ${ }^{9}$

Annotation Process The annotation process was as follows: Firstly, the annotators conducted discussions about the conflicting annotations after several attempts to annotate the same three scripts. Secondly, once agreements about how to solve the conflicts had been reached, they started to annotate their assigned scripts. Afterwards, since information extraction annotation is more complex than that of classification annotations, we measured its quality by computing three types of interannotator agreement. We asked the annotators to annotate the same six scripts having different styles and then calculated the Overall Percent Agreement (OPA), Fleiss's kappa (Fleiss, 1971) and Randolph's kappa (Randolph, 2005). We found that the agreement rates were high (OPA 98.09\%, Fleiss's Kappa 0.85, Randolph's Kappa 0.96). This is due to the fact that the discussions about solving conflicts were in-depth and the laughter triggers were usually clear in the lines.

\section{Baselines and Results Discussion}

In order to understand how well the machine learning methods work on our corpus, we evaluate the performances of a number of models on 5-fold cross-validation random splits of the scripts in our corpus and report the average results in this section. ${ }^{10}$ All the BERT models were pre-trained by using a mixture of large Chinese corpora. ${ }^{11}$ Please

\footnotetext{
${ }^{9}$ The house is actually small. Since there is almost no furniture in the house, the comedian said it looked big.

${ }^{10}$ Model implementations were adapted from https://github.com/649453932/ Chinese-Text-Classification-Pytorch, https: //github.com/luopeixiang/named_entity_ recognition and Zhao et al. (2019).

${ }^{11}$ More details are listed in https://github.com/ dbiir/UER-py/wiki/Modelzoo.
}

\begin{tabular}{lcccc}
\hline Model & P & R & F & Acc. \\
\hline CNN (Kim, 2014) & 42.53 & 64.14 & 51.07 & 66.29 \\
RCNN (Lai et al., 2015) & 41.21 & $\mathbf{6 8 . 5 2}$ & 50.89 & 63.54 \\
BiLSTM (Liu et al., 2016) & 41.17 & 57.13 & 47.66 & 65.69 \\
+ Attention (Zhou et al., 2016) & 39.97 & 59.91 & 47.44 & 63.94 \\
FastText (Joulin et al., 2017) & 40.61 & 66.26 & 50.12 & 63.72 \\
DPCNN (Johnson and Zhang, 2017) & 42.46 & 63.25 & 50.76 & 66.32 \\
Transformer (Vaswani et al., 2017) & 42.60 & 64.71 & 51.24 & 66.18 \\
BERT-tiny (Jiao et al., 2019) & 47.56 & 53.38 & 48.91 & 66.21 \\
BERT-small (Turc et al., 2019) & 47.29 & 56.21 & 51.21 & 70.78 \\
BERT-base (Devlin et al., 2019) & $\mathbf{4 7 . 6 0}$ & 56.64 & $\mathbf{5 1 . 6 1}$ & $\mathbf{7 0 . 9 4}$ \\
\hline
\end{tabular}

Table 5: Text classification performance. $\boldsymbol{P}, \boldsymbol{R}, \boldsymbol{F}$ and Acc. are Precision, Recall, F1-score and Accuracy respectively.

\begin{tabular}{lccc}
\hline Model & P & R & F \\
\hline HMM (Rabiner and Juang, 1986) & 22.19 & 7.43 & 11.04 \\
CRF (Lafferty et al., 2001) & 28.56 & 6.11 & 10.01 \\
BiLSTM (Huang et al., 2015) & $\mathbf{3 1 . 2 1}$ & 1.64 & 3.09 \\
BiLSTM-CRF (Lample et al., 2016) & 30.33 & 9.81 & 14.48 \\
BERT-tiny (Jiao et al., 2019) & 26.26 & 19.89 & 22.57 \\
BERT-small (Turc et al., 2019) & 28.82 & 17.52 & 21.56 \\
BERT-base (Devlin et al., 2019) & 30.15 & $\mathbf{2 1 . 4 7}$ & $\mathbf{2 4 . 5 9}$ \\
\hline
\end{tabular}

Table 6: Information extraction performance. Relaxed metrics are used. The exact-match metric is over-strict because the length of text spans in this corpus is much longer than general named entities. The computation of these metrics can be found in (Nguyen et al., 2017).

refer to the appendix for the results of each fold, statistics of splits, computing infrastructure, each model's running time, parameter details and hyperparameter settings.

Baselines Tables 5 and 6 respectively present the results of text classification and information extraction. BERT-base has the best F1-scores among all the methods. We also note that the classification recall of RCNN (Lai et al., 2015) is much higher than other methods. Therefore, we suggest using this model if users prefer a classifier with a high recall. In addition, we observe that the scores are not high, especially for the information extraction task. The reason may be if the audience laughter highly depends on the conversation contexts which were not considered by baselines. Therefore, taking a longer conversation context of a line into consideration is a worthy research direction.

Prediction Errors Tables 7 and 8 present examples incorrectly predicted by the BERT-base. The first 3 examples describe how the model failed to predict the laughter triggers, while the last 3 examples show false positive predictions. Incorporating the context information of lines may further reduce these errors.

Cross-Style Performance We further investigate the performance of predicting laughter triggers on scripts with a totally different style compared to 


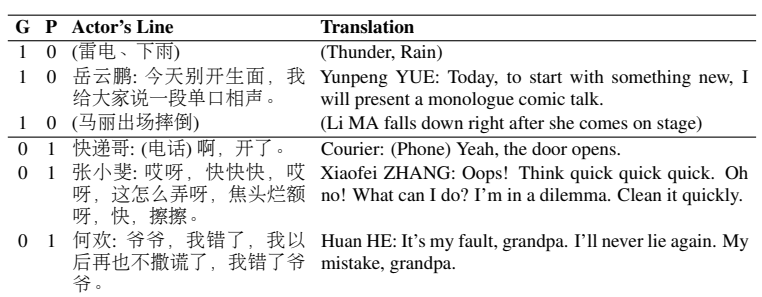

Table 7: Examples of incorrect classifications taken from different comedies in our corpus. $\boldsymbol{G}$ and $\boldsymbol{P}$ are Gold and Predicted labels, respectively.

\begin{tabular}{|c|c|}
\hline Actor's Line & Translation \\
\hline $\begin{array}{l}\text { 贾冰: 这事我不敢保证我现在 } \\
\text { 还认不[认字啊]。 }\end{array}$ & $\begin{array}{l}\text { Bing JIA: I can't guarantee if I am still [able to read } \\
\text { this] now. }\end{array}$ \\
\hline 岳云鹏：你什么时候来的啊。 & Yuepeng YUE: Huh? When did you come? \\
\hline $\begin{array}{l}\text { 沈腾: 走肯定是不赶趟了, 我 } \\
\text { 得跑了。 }\end{array}$ & $\begin{array}{l}\text { Teng SHEN: Walking is not fast enough, I have to } \\
\text { run to escape this. }\end{array}$ \\
\hline 快递哥: [散打那个]。 & Courier: [The one who is good at free combat]. \\
\hline $\begin{array}{l}\text { 张小斐: 䒝呀, 这是善意的谎 } \\
\text { 言, 可问题是, 老师也不会[演 } \\
\text { 戏呀]! }\end{array}$ & $\begin{array}{l}\text { Xiaofei ZHANG: Well, this is a white lie. But the } \\
\text { problem is, I don't know how [to pretend]! }\end{array}$ \\
\hline 何欢: 不是, 你[找谁呀]? & Huan HE: Eh? [Who are] you [looking for]? \\
\hline
\end{tabular}

Table 8: Examples of incomplete and incorrect extractions taken from different comedies in our corpus. Characters in bold and located in [ ] are the gold annotations and predicted results respectively.

the styles in the training data. Firstly, since the six comedians in the corpus have distinctive comedy styles, we split the entire corpus into a 6-fold crossvalidation manner. The comedies in each fold are performed by the same leading comedian. Secondly, we train baseline models on five of the folds and evaluate the performance on the remaining fold. Tables 9 and 10 present the average results and the full results are available in the appendix. The results demonstrate that the laughter triggers can be detected even though the styles in the training data are very different compared to the testing data.

\section{Conclusion and Future Work}

We study the prediction of laughter triggers from comedy scripts by using text classification and information extraction methods. Firstly, we introduce a corpus including high-quality and annotated sketch comedy and crosstalk scripts. Secondly, we evaluate a number of baselines and find that BERT models achieve the best performance. We note that the information extraction performance was very low, indicating that this task is particularly challenging. We also conduct an error analysis of incorrect predictions. The errors suggest the incorporation of rich context information may further improve the performance. Therefore, it is worth investigating a model which can take such infor-

\begin{tabular}{lcccc}
\hline Model & P & R & F & Acc. \\
\hline CNN (Kim, 2014) & 42.75 & 65.69 & 51.63 & 65.04 \\
RCNN (Lai et al., 2015) & 42.32 & $\mathbf{7 1 . 2 6}$ & $\mathbf{5 2 . 3 2}$ & 62.79 \\
BiLSTM (Liu et al., 2016) & 41.35 & 60.76 & 48.81 & 63.71 \\
+ Attention (Zhou et al., 2016) & 43.20 & 53.76 & 47.57 & 66.55 \\
FastText (Joulin et al., 2017) & 40.86 & 67.43 & 50.70 & 62.62 \\
DPCNN (Johnson and Zhang, 2017) & 41.26 & 66.82 & 50.42 & 62.44 \\
Transformer (Vaswani et al., 2017) & 41.57 & 70.15 & 51.92 & 63.06 \\
BERT-tiny (Jiao et al., 2019) & 43.01 & 56.76 & 48.69 & 66.23 \\
BERT-small (Turc et al., 2019) & 44.95 & 55.59 & 49.09 & 67.38 \\
BERT-base (Devlin et al., 2019) & $\mathbf{4 7 . 2 8}$ & 58.13 & 51.72 & $\mathbf{6 9 . 3 9}$ \\
\hline
\end{tabular}

Table 9: Cross-style text classification performance.

\begin{tabular}{lccc}
\hline Model & P & R & F \\
\hline HMM (Rabiner and Juang, 1986) & 19.79 & 7.52 & 10.68 \\
CRF (Lafferty et al., 2001) & 25.29 & 5.03 & 8.35 \\
BiLSTM (Huang et al., 2015) & 29.05 & 2.90 & 5.23 \\
BiLSTM-CRF (Lample et al., 2016) & $\mathbf{3 0 . 7 2}$ & 8.77 & 12.56 \\
BERT-tiny (Jiao et al., 2019) & 26.30 & 19.20 & 22.14 \\
BERT-small (Turc et al., 2019) & 24.93 & 27.31 & 25.12 \\
BERT-base (Devlin et al., 2019) & 24.64 & $\mathbf{3 1 . 6 5}$ & $\mathbf{2 6 . 5 1}$ \\
\hline
\end{tabular}

Table 10: Cross-style information extraction performance.

mation into consideration. Furthermore, it is also worth extending the corpus to a multimodal one by aligning scripts to corresponding audios or videos, because certain intonations or scenes can also make audiences laugh. The multimodal corpus can also benefit the creation of silent comedy. Enriching the corpus by including scripts in other languages may also be a potential direction. Lastly, the encouraging cross-style prediction performance shows the usefulness of our corpus for predicting new scripts with different styles.

Moreover, it is also interesting to explore human performances by asking annotators to make predictions based purely on the scripts of unwatched comedies, and to investigate if the script writers find the model predictions insightful.

We hope this study will benefit script writing by inspiring the community to develop intelligent systems for comedy writers and other artists in the field. The corpus might also be useful for researchers who are working on related or similar tasks, such as discourse analysis and humorous response generation for dialogue systems.

\section{Acknowledgements}

We would like to express our sincere appreciation to all comedians and backstage teams for their hard work to make audiences happy. We also sincerely appreciate the valuable comments of all anonymous reviewers. My deepest thanks should also be given to my best friend who inspired me to conduct such research and whose encouragement was important for me to be able to complete the work. 


\section{References}

Dario Bertero. 2019. Conversational humor recognition and generation through deep learning. $\mathrm{PhD}$ dissertation, Hong Kong University of Science and Technology.

Marc Blake. 2014. How to be a Comedy Writer, volume 1. Andrews UK Limited.

Vladislav Blinov, Valeria Bolotova-Baranova, and Pavel Braslavski. 2019. Large dataset and language model fun-tuning for humor recognition. In Proceedings of the 57th Annual Meeting of the Association for Computational Linguistics, pages 40274032, Florence, Italy. Association for Computational Linguistics.

Ernesto Carrera-Ruvalcaba, Johnson Ekedum, Austin Hancock, and Ben Brock. 2019. Leveraging natural language processing applications and microblogging platform for increased transparency in crisis areas. SMU Data Science Review, 2(1):6.

Santiago Castro, Luis Chiruzzo, Aiala Rosá, Diego Garat, and Guillermo Moncecchi. 2018. A crowdannotated Spanish corpus for humor analysis. In Proceedings of the Sixth International Workshop on Natural Language Processing for Social Media, pages 7-11, Melbourne, Australia. Association for Computational Linguistics.

Peng-Yu Chen and Von-Wun Soo. 2018. Humor recognition using deep learning. In Proceedings of the 2018 Conference of the North American Chapter of the Association for Computational Linguistics: $\mathrm{Hu}$ man Language Technologies, Volume 2 (Short Papers), pages 113-117, New Orleans, Louisiana. Association for Computational Linguistics.

Luis Chiruzzo, S Castro, Mathias Etcheverry, Diego Garat, Juan José Prada, and Aiala Rosá. 2019. Overview of haha at iberlef 2019: Humor analysis based on human annotation. In Proceedings of the Iberian Languages Evaluation Forum (IberLEF 2019). CEUR Workshop Proceedings, CEURWS, Bilbao, Spain.

Jacob Devlin, Ming-Wei Chang, Kenton Lee, and Kristina Toutanova. 2019. BERT: Pre-training of deep bidirectional transformers for language understanding. In Proceedings of the 2019 Conference of the North American Chapter of the Association for Computational Linguistics: Human Language Technologies, Volume 1 (Long and Short Papers), pages 4171-4186, Minneapolis, Minnesota. Association for Computational Linguistics.

Joseph L Fleiss. 1971. Measuring nominal scale agreement among many raters. Psychometrika, 76(5):378.

Md Kamrul Hasan, Wasifur Rahman, AmirAli Bagher Zadeh, Jianyuan Zhong, Md Iftekhar Tanveer, Louis-Philippe Morency, and Mohammed (Ehsan) Hoque. 2019. UR-FUNNY:
A multimodal language dataset for understanding humor. In Proceedings of the 2019 Conference on Empirical Methods in Natural Language Processing and the 9th International Joint Conference on Natural Language Processing (EMNLP-IJCNLP), pages 2046-2056, Hong Kong, China. Association for Computational Linguistics.

Zhiheng Huang, Wei Xu, and Kai Yu. 2015. Bidirectional lstm-crf models for sequence tagging. arXiv preprint arXiv:1508.01991.

Xiaoqi Jiao, Yichun Yin, Lifeng Shang, Xin Jiang, Xiao Chen, Linlin Li, Fang Wang, and Qun Liu. 2019. Tinybert: Distilling bert for natural language understanding. arXiv preprint arXiv:1909.10351.

Rie Johnson and Tong Zhang. 2017. Deep pyramid convolutional neural networks for text categorization. In Proceedings of the 55th Annual Meeting of the Association for Computational Linguistics (Volume 1: Long Papers), pages 562-570, Vancouver, Canada. Association for Computational Linguistics.

Armand Joulin, Edouard Grave, Piotr Bojanowski, and Tomas Mikolov. 2017. Bag of tricks for efficient text classification. In Proceedings of the 15th Conference of the European Chapter of the Association for Computational Linguistics: Volume 2, Short Papers, pages 427-431, Valencia, Spain. Association for Computational Linguistics.

Yoon Kim. 2014. Convolutional neural networks for sentence classification. In Proceedings of the 2014 Conference on Empirical Methods in Natural Language Processing (EMNLP), pages 1746-1751, Doha, Qatar. Association for Computational Linguistics.

John Lafferty, Andrew McCallum, and Fernando CN Pereira. 2001. Conditional random fields: Probabilistic models for segmenting and labeling sequence data. In Proceedings of the 18th International Conference on Machine Learning, San Francisco, CA, USA. Morgan Kaufmann Publishers Inc.

Siwei Lai, Liheng Xu, Kang Liu, and Jun Zhao. 2015. Recurrent convolutional neural networks for text classification. In Proceedings of the TwentyNinth AAAI Conference on Artificial Intelligence, AAAI'15, page 2267-2273. AAAI Press.

Guillaume Lample, Miguel Ballesteros, Sandeep Subramanian, Kazuya Kawakami, and Chris Dyer. 2016. Neural architectures for named entity recognition. In Proceedings of the 2016 Conference of the North American Chapter of the Association for Computational Linguistics: Human Language Technologies, pages 260-270, San Diego, California. Association for Computational Linguistics.

Da Li, Rafal Rzepka, Michal Ptaszynski, and Kenji Araki. 2020. Hemos: A novel deep learning-based fine-grained humor detecting method for sentiment analysis of social media. Information Processing \& Management, 57(6):102290. 
Pengfei Liu, Xipeng Qiu, and Xuanjing Huang. 2016. Recurrent neural network for text classification with multi-task learning. In Proceedings of the TwentyFifth International Joint Conference on Artificial Intelligence, IJCAI'16, page 2873-2879. AAAI Press.

Rada Mihalcea and Carlo Strapparava. 2006. Learning to laugh (automatically): Computational models for humor recognition. Computational Intelligence, 22(2):126-142.

Hiroki Nakayama, Takahiro Kubo, Junya Kamura, Yasufumi Taniguchi, and Xu Liang. 2018. doccano: Text annotation tool for human. Software available from https://github.com/doccano/doccano.

An Thanh Nguyen, Byron Wallace, Junyi Jessy Li, Ani Nenkova, and Matthew Lease. 2017. Aggregating and predicting sequence labels from crowd annotations. In Proceedings of the 55th Annual Meeting of the Association for Computational Linguistics (Volume 1: Long Papers), pages 299-309, Vancouver, Canada. Association for Computational Linguistics.

Reynier Ortega-Bueno, Carlos E Muniz-Cuza, José E Medina Pagola, and Paolo Rosso. 2018. Uo upv: Deep linguistic humor detection in spanish social media. In Proceedings of the Third Workshop on Evaluation of Human Language Technologies for Iberian Languages (IberEval 2018) co-located with 34th Conference of the Spanish Society for Natural Language Processing (SEPLN 2018), pages 203 213, Seville, Spain.

Lawrence Rabiner and B Juang. 1986. An introduction to hidden markov models. IEEE ASSP Magazine, 3(1):4-16.

JJ Randolph. 2005. Free-marginal multirater kappa: an alternative to fleiss' fixed-marginal multirater kappa. Joensuu University Learning and Instruction Symposium.

Delip Rao and Brian McMahan. 2019. Natural language processing with PyTorch: build intelligent language applications using deep learning. " O’Reilly Media, Inc.”.

Serdar Sarıtaş, Hasan Genç, Şerafettin Okutan, Ramazan İnci, Ahmet Özdemir, and Gülnaz Kizilkaya. 2019. The effect of comedy films on postoperative pain and anxiety in surgical oncology patients. Complementary Medicine Research, 26(4):231-239.

Iulia Turc, Ming-Wei Chang, Kenton Lee, and Kristina Toutanova. 2019. Well-read students learn better: The impact of student initialization on knowledge distillation. arXiv preprint arXiv:1908.08962.

Ashish Vaswani, Noam Shazeer, Niki Parmar, Jakob Uszkoreit, Llion Jones, Aidan N Gomez, Ł ukasz Kaiser, and Illia Polosukhin. 2017. Attention is all you need. In I. Guyon, U. V. Luxburg, S. Bengio, H. Wallach, R. Fergus, S. Vishwanathan, and R. Garnett, editors, Advances in Neural Information Processing Systems 30, pages 5998-6008. Curran Associates, Inc.
Sandy M Williams, Pauline K Arnold, and Jennifer N Mills. 2005. Coping with stress: a survey of murdoch university veterinary students. Journal of Veterinary Medical Education, 32(2):201-212.

Diyi Yang, Alon Lavie, Chris Dyer, and Eduard Hovy. 2015. Humor recognition and humor anchor extraction. In Proceedings of the 2015 Conference on Empirical Methods in Natural Language Processing, pages 2367-2376, Lisbon, Portugal. Association for Computational Linguistics.

Dongyu Zhang, Heting Zhang, Xikai Liu, Hongfei Lin, and Feng Xia. 2019. Telling the whole story: A manually annotated Chinese dataset for the analysis of humor in jokes. In Proceedings of the 2019 Conference on Empirical Methods in Natural Language Processing and the 9th International Joint Conference on Natural Language Processing (EMNLPIJCNLP), pages 6402-6407, Hong Kong, China. Association for Computational Linguistics.

Renxian Zhang and Naishi Liu. 2014. Recognizing humor on twitter. In Proceedings of the 23rd ACM International Conference on Conference on Information and Knowledge Management, CIKM '14, page 889-898, New York, NY, USA. Association for Computing Machinery.

Zhe Zhao, Hui Chen, Jinbin Zhang, Xin Zhao, Tao Liu, Wei Lu, Xi Chen, Haotang Deng, Qi Ju, and Xiaoyong Du. 2019. UER: An open-source toolkit for pretraining models. In Proceedings of the 2019 Conference on Empirical Methods in Natural Language Processing and the 9th International Joint Conference on Natural Language Processing (EMNLPIJCNLP): System Demonstrations, pages 241-246, Hong Kong, China. Association for Computational Linguistics.

Peng Zhou, Wei Shi, Jun Tian, Zhenyu Qi, Bingchen Li, Hongwei Hao, and Bo Xu. 2016. Attention-based bidirectional long short-term memory networks for relation classification. In Proceedings of the 54th Annual Meeting of the Association for Computational Linguistics (Volume 2: Short Papers), pages 207-212, Berlin, Germany. Association for Computational Linguistics.

\section{A Appendices}

\section{A.1 Computing Resources}

Table 11 describes the details of the computing resources used for all of our experiments. These resources are freely available from Paperspace ${ }^{12}$.

\section{A.2 Model Details}

Below we present model hyper-parameter values $^{13}$ and the average running time of one epoch.

\footnotetext{
${ }^{12}$ https: / / www . paperspace.com/

${ }^{13}$ The size of model's trainable parameters can be found in original papers.
} 


\begin{tabular}{ll}
\hline Name & Description \\
\hline CPU & 8 vCPUs, 30GB RAM \\
GPU & NVIDIA Quadro P5000 \\
& Graphics Card, 16GB \\
Processor & Intel Xeon \\
Clock Speed & $2.60 \mathrm{GHz}$ \\
\hline
\end{tabular}

Table 11: Details of computing resources.

\section{A.2.1 Text Classification Models}

CNN (Kim, 2014) Dropout $=0.5$, Number of Epochs $=20$, Batch Size $=128$, Learning Rate $=$ 0.001, Number of Filters $=256$, Filter Sizes $=2,3,4$, Average Running Time $=5 \mathrm{~s}$.

RCNN (Lai et al., 2015) Dropout $=1.0$, Number of Epochs $=10$, Batch Size $=128$, Learning Rate $=0.001$, Hidden Size $=256$, Number of Layers $=$ 1, Average Running Time $=5 \mathrm{~s}$.

BiLSTM (Liu et al., 2016) Dropout $=0.5$, Number of Epochs $=10$, Batch Size = 128, Learning Rate $=0.001$, Hidden Size $=128$, Number of Layers $=2$, Average Running Time $=5.4 \mathrm{~s}$.

+ Attention (Zhou et al., 2016) Dropout $=0.5$, Number of Epochs $=10$, Batch Size $=128$, Learning Rate $=0.001$, Hidden Size $=128$ and 64 respectively, Number of Layers $=2$, Average Running Time $=5.74 \mathrm{~s}$.

FastText (Joulin et al., 2017) Dropout $=0.5$, Number of Epochs $=20$, Batch Size $=128$, Learning Rate $=0.001$, Hidden Size $=256$, Average Running Time $=22.5 \mathrm{~s}$.

DPCNN (Johnson and Zhang, 2017) Dropout $=0.5$, Number of Epochs $=20$, Batch Size $=128$, Learning Rate $=0.001$, Number of Filter $=250$, Average Running Time $=5 \mathrm{~s}$.

Transformer (Vaswani et al., 2017) Dropout $=$ 0.5, Number of Epochs $=20$, Batch Size $=128$, Learning Rate $=0.0005$, Number of Head $=5$, Number of Encoder $=2$, Average Running Time $=$ $6.528 \mathrm{~s}$.

BERT-tiny (Jiao et al., 2019) Dropout $=0.1$, Number of Epoch $=20$, Batch Size $=64$, Learning Rate $=0.00002$, Size of Embedding $=384$, Feedforward Size $=1536$, Hidden Size $=384$, Number of Head $=6$, Number of Layer $=3$, Average Running Time $=20.63 \mathrm{~s}$.
BERT-small (Turc et al., 2019) Dropout $=0.5$, Number of Epoch $=20$, Batch Size $=64$, Learning Rate $=0.00002$, Size of Embedding $=512$, Feedforward Size $=2048$, Hidden Size $=512$, Number of Head $=8$, Number of Layer $=6$, Average Running Time $=36.65$.

BERT-base (Devlin et al., 2019) Dropout $=0.1$, Number of Epoch $=10$, Batch Size $=64$, Learning Rate $=0.00002$, Size of Embedding $=768$, Feedforward Size $=3072$, Hidden Size $=768$, Number of Head $=12$, Number of Layer $=12$, Average Running Time $=99 \mathrm{~s}$.

\section{A.2.2 Information Extraction Models}

HMM (Rabiner and Juang, 1986) Uniform Distribution for Initialisation, Average Total Running Time $=8.47 \mathrm{~s}$.

CRF (Lafferty et al., 2001) LBFGS algorithm, $\mathrm{c} 1=0.1, \mathrm{c} 2=0.1$, Max Iteration $=100$, Average Total Running Time $=11.72 \mathrm{~s}$.

BiLSTM (Huang et al., 2015) Number of Epoch $=30$, Batch Size $=64$, Learning Rate $=$ 0.001, Size of Embedding $=128$, Hidden Size $=$ 128, Average Running Time $=8.43 \mathrm{~s}$.

BiLSTM-CRF (Lample et al., 2016) Number of Epoch $=30$, Batch Size $=64$, Learning Rate $=$ 0.001, Size of Embedding $=128$, Hidden Size $=$ 128, Average Running Time $=9.35 \mathrm{~s}$.

BERT Models We use the same hyper-parameter settings as used in the text classification models with the exception of Batch Size $=16$. The average running time of BERT-tiny, BERT-small and BERTbase for information extraction are $34.00 \mathrm{~s}, 55.73 \mathrm{~s}$, and 120 s respectively.

\section{A.3 Details of Baseline Experiments}

Table 12 shows the statistics of each fold.

\begin{tabular}{ccccc}
\hline \multirow{2}{*}{ Fold } & \multicolumn{2}{c}{ Text Classification } & \multicolumn{2}{c}{ Information Extraction } \\
& \# of Lines & Total & \# of Characters & Total \\
\hline 0 & $277 / 685$ & 962 & $1666 / 20010$ & 21676 \\
1 & $290 / 854$ & 1144 & $1703 / 19354$ & 21057 \\
2 & $285 / 789$ & 1074 & $1666 / 19609$ & 21275 \\
3 & $358 / 909$ & 1267 & $2251 / 23535$ & 25786 \\
4 & $459 / 1181$ & 1640 & $2632 / 28025$ & 30657 \\
\hline
\end{tabular}

Table 12: Statistics of each fold in the baseline experiments. The number before slash indicates how many actor's lines or characters that make the audience laugh. The number after slash indicates the number of lines or characters without causing audiences laugh. 


\begin{tabular}{|c|c|c|c|c|c|c|c|}
\hline & Model & Fold-0 & Fold-1 & Fold-2 & Fold-3 & Fold-4 & Average \\
\hline & CNN & 42.64 & 40.43 & 42.61 & 42.27 & 44.68 & 42.53 \\
\hline & RCNN & 43.47 & 38.30 & 43.93 & 35.44 & 44.93 & 41.21 \\
\hline & BiLSTM & 43.56 & 37.80 & 40.19 & 42.20 & 42.12 & 41.17 \\
\hline & +Attention & 39.53 & 38.93 & 41.75 & 37.62 & 42.01 & 39.97 \\
\hline & FastText & 39.37 & 44.64 & 36.96 & 41.12 & 40.98 & 40.61 \\
\hline & DPCNN & 42.44 & 41.67 & 41.73 & 41.86 & 44.61 & 42.46 \\
\hline & Transformer & 46.67 & 38.17 & 42.43 & 42.08 & 43.66 & 42.60 \\
\hline & BERT-tiny & 42.26 & 42.05 & 44.62 & 54.43 & 54.43 & 47.56 \\
\hline & BERT-small & 44.74 & 46.13 & 46.02 & 52.35 & 47.22 & 47.29 \\
\hline & BERT-base & 45.65 & 47.73 & 42.66 & 53.03 & 48.91 & 47.60 \\
\hline \multirow{10}{*}{$\mathbf{R}$} & $\mathrm{CNN}$ & 70.04 & 64.02 & 60.70 & 66.48 & 59.48 & 64.14 \\
\hline & RCNN & 66.06 & 70.08 & 59.65 & 86.03 & 60.78 & 68.52 \\
\hline & BiLSTM & 51.26 & 65.15 & 58.95 & 56.70 & 53.59 & 57.13 \\
\hline & +Attention & 55.23 & 65.91 & 58.60 & 75.14 & 44.66 & 59.91 \\
\hline & FastText & 68.23 & 56.82 & 71.58 & 63.41 & 71.24 & 66.26 \\
\hline & DPCNN & 67.87 & 62.50 & 61.05 & 65.36 & 59.48 & 63.25 \\
\hline & Transformer & 60.65 & 69.70 & 64.91 & 62.29 & 66.01 & 64.71 \\
\hline & BERT-tiny & 62.09 & 41.03 & 59.65 & 49.72 & 54.43 & 53.38 \\
\hline & BERT-small & 59.93 & 47.24 & 62.81 & 59.22 & 51.85 & 56.21 \\
\hline & BERT-base & 62.45 & 50.69 & 55.09 & 61.17 & 53.81 & 56.64 \\
\hline \multirow{10}{*}{$\mathbf{F}$} & $\mathrm{CNN}$ & 53.01 & 49.56 & 50.07 & 51.68 & 51.03 & 51.07 \\
\hline & RCNN & 52.44 & 49.53 & 50.60 & 50.20 & 51.67 & 50.89 \\
\hline & BiLSTM & 47.10 & 47.84 & 47.80 & 48.39 & 47.17 & 47.66 \\
\hline & +Attention & 46.08 & 48.95 & 48.76 & 50.14 & 43.29 & 47.44 \\
\hline & FastText & 49.93 & 50.00 & 48.75 & 49.89 & 52.03 & 50.12 \\
\hline & DPCNN & 52.22 & 50.00 & 49.57 & 51.04 & 50.98 & 50.76 \\
\hline & Transformer & 52.75 & 49.33 & 51.32 & 50.23 & 52.56 & 51.24 \\
\hline & BERT-tiny & 50.29 & 41.54 & 51.05 & 51.97 & 49.72 & 48.91 \\
\hline & BERT-small & 51.23 & 46.68 & 53.12 & 55.57 & 49.43 & 51.21 \\
\hline & BERT-base & 52.74 & 49.16 & 48.09 & 56.81 & 51.24 & 51.61 \\
\hline \multirow{10}{*}{ A } & $\mathrm{CNN}$ & 64.24 & 66.41 & 67.88 & 64.88 & 68.05 & 66.29 \\
\hline & RCNN & 65.49 & 63.18 & 69.09 & 51.78 & 68.17 & 63.54 \\
\hline & BiLSTM & 66.84 & 63.38 & 65.83 & 65.82 & 66.40 & 65.65 \\
\hline & +Attention & 62.79 & 64.55 & 67.32 & 57.77 & 67.26 & 63.94 \\
\hline & FastText & 60.60 & 70.70 & 60.06 & 64.01 & 63.23 & 63.72 \\
\hline & DPCNN & 64.24 & 67.77 & 67.04 & 64.56 & 67.99 & 66.32 \\
\hline & Transformer & 68.71 & 63.09 & 67.32 & 65.11 & 66.65 & 66.18 \\
\hline & BERT-tiny & 64.66 & 70.72 & 69.65 & 74.03 & 51.97 & 66.21 \\
\hline & BERT-small & 67.15 & 72.64 & 70.58 & 73.24 & 70.30 & 70.78 \\
\hline & BERT-base & 67.78 & 73.43 & 68.44 & 73.72 & 71.34 & 70.94 \\
\hline
\end{tabular}

Table 13: Each fold's text classification baseline experiments and their overall average performance. $\boldsymbol{P}, \boldsymbol{R}, \boldsymbol{F}$ and $\boldsymbol{A}$ are Precision, Recall, F1-score and Accuracy respectively.

Tables 13 and 14 describe the detailed performance of text classification and information extraction baseline experiments.

\begin{tabular}{llcccccc}
\hline Model & Fold-0 & Fold-1 & Fold-2 & Fold-3 & Fold-4 & Average \\
\hline & HMM & 18.26 & 21.15 & 26.37 & 19.46 & 25.70 & 22.19 \\
CRF & 24.62 & $\mathbf{3 0 . 8 6}$ & $\mathbf{3 4 . 1 7}$ & 23.33 & 29.83 & 28.56 \\
BILSTM & 26.25 & 37.14 & 26.00 & $\mathbf{3 7 . 3 1}$ & 29.35 & $\mathbf{3 1 . 2 1}$ \\
P & BiLSTM-CRF & 30.50 & 24.43 & 32.46 & 29.71 & $\mathbf{3 4 . 5 6}$ & 30.33 \\
& BERT-tiny & 27.64 & 23.98 & 28.89 & 26.73 & 24.08 & 26.26 \\
BERT-small & 31.71 & 24.12 & 30.83 & 28.04 & 29.38 & 28.82 \\
BERT-base & $\mathbf{3 6 . 2 3}$ & 28.16 & 26.56 & 30.88 & 28.93 & 30.15 \\
\hline HMM & 7.19 & 9.64 & 7.38 & 5.93 & 7.02 & 7.43 \\
& CRF & 6.49 & 5.49 & 6.32 & 6.47 & 5.76 & 6.11 \\
BILSTM & 2.24 & 1.23 & 0.55 & 2.47 & 1.70 & 1.64 \\
R & BiLSTM-CRF & 16.76 & 8.00 & 9.25 & 6.30 & 8.73 & 9.81 \\
& BERT-tiny & 17.37 & 19.54 & 20.96 & $\mathbf{2 2 . 4 6}$ & 19.10 & 19.89 \\
& BERT-small & 16.90 & 19.22 & 16.26 & 21.35 & 13.86 & 17.52 \\
& BERT-base & $\mathbf{2 1 . 6 2}$ & $\mathbf{2 1 . 1 9}$ & $\mathbf{2 7 . 1 7}$ & 21.02 & $\mathbf{1 6 . 3 5}$ & $\mathbf{2 1 . 4 7}$ \\
\hline & HMM & 10.32 & 13.24 & 11.53 & 9.09 & 11.03 & 11.04 \\
CRF & 10.27 & 9.33 & 10.67 & 10.13 & 9.65 & 10.01 \\
BILSTM & 4.13 & 2.37 & 1.08 & 4.63 & 3.22 & 3.09 \\
F & BiLSTM-CRF & 21.63 & 12.05 & 14.40 & 10.39 & 13.94 & 14.48 \\
& BERT-tiny & 21.33 & 21.53 & 24.30 & 24.41 & $\mathbf{2 1 . 3 0}$ & 2.57 \\
BERT-small & 22.05 & 21.40 & 21.29 & 24.24 & 18.83 & 2.56 \\
BERT-base & $\mathbf{2 6 . 0 1}$ & $\mathbf{2 4 . 1 8}$ & $\mathbf{2 6 . 8 6}$ & $\mathbf{2 5 . 0 2}$ & 20.89 & $\mathbf{2 4 . 5 9}$ \\
\hline
\end{tabular}

Table 14: Each fold's information extraction baseline experiments and their overall average performance.

\section{A.4 Details of Cross-Style Experiments}

Table 15 shows the statistics of the scripts performed by specific leading comedians. Tables 16 and 17 present the prediction results.

\begin{tabular}{lcccc}
\hline \multirow{2}{*}{ Comedian } & \multicolumn{2}{c}{ Text Classification } & \multicolumn{2}{c}{ Information Extraction } \\
& \# of Lines & Total & \# of Characters & Total \\
\hline Xiaobao SONG & $315 / 815$ & 1150 & $2001 / 20366$ & 22367 \\
Yuepeng YUE & $436 / 1547$ & 1983 & $2362 / 25579$ & 27941 \\
Ling JIA & $195 / 501$ & 696 & $1135 / 14414$ & 15549 \\
Xiaofei ZHANG & $190 / 495$ & 685 & $1056 / 15946$ & 17002 \\
Teng SHEN & $166 / 350$ & 516 & $1071 / 13143$ & 14214 \\
Bing JIA & $367 / 690$ & 1057 & $2293 / 21085$ & 23378 \\
\hline
\end{tabular}

Table 15: Statistics of the scripts performed by specific leading comedians.

\section{A.5 Full List of Selected Comedy Performances}

Tables 18 and 19 show the full list of performances in our corpus with details. 


\begin{tabular}{|c|c|c|c|c|c|c|c|c|}
\hline & Model & $\mathbf{0}$ & 1 & 2 & 3 & 4 & 5 & Average \\
\hline \multirow{10}{*}{$\mathbf{F}$} & $\mathrm{CNN}$ & 41.99 & 40.00 & 41.85 & 39.67 & 45.70 & 47.29 & 42.75 \\
\hline & BiLSTM & 38.17 & 42.18 & 39.35 & 36.26 & 45.26 & 46.90 & 41.35 \\
\hline & + Attention & 42.70 & 38.16 & 45.06 & 38.49 & 48.02 & 46.78 & 43.20 \\
\hline & RCNN & 46.93 & 44.01 & 36.36 & 37.23 & 46.26 & 43.10 & 42.32 \\
\hline & FastText & 41.88 & 37.99 & 36.64 & 37.81 & 45.24 & 45.57 & 40.86 \\
\hline & DPCNN & 42.01 & 42.21 & 36.03 & 35.25 & 45.33 & 46.71 & 41.26 \\
\hline & Transformer & 41.97 & 36.32 & 36.94 & 41.30 & 46.50 & 46.41 & 41.57 \\
\hline & BERT-tiny & 40.73 & 37.41 & 42.38 & 40.81 & 45.30 & 51.40 & 43.01 \\
\hline & BERT-small & 39.08 & 39.67 & 42.97 & 48.40 & 43.22 & 56.38 & 95 \\
\hline & BERT-base & 42.50 & 44.40 & 42.73 & 48.68 & 48.05 & 57.31 & 47.28 \\
\hline \multirow{10}{*}{ F } & $\mathrm{CNN}$ & 61.59 & 51.38 & 69.74 & 66.48 & 80.61 & 64.31 & 65.69 \\
\hline & BiLST & 51.75 & 47.02 & 62.56 & 70.33 & 75.15 & 57.77 & 76 \\
\hline & + Attention & 36.19 & 55.05 & 53.85 & 53.30 & 58.79 & 65.40 & 76 \\
\hline & RCNN & 55.87 & 51.38 & 82.05 & 75.27 & 78.79 & 84.20 & 26 \\
\hline & FastText & 62.22 & 55.50 & 73.85 & 66.48 & 80.61 & 65.94 & 67.43 \\
\hline & DPCNN & 60.95 & 47.25 & 75.38 & 74.18 & 79.39 & 63.76 & 82 \\
\hline & Transformer & 62.22 & 62.39 & 84.10 & 66.48 & 80.61 & 65.12 & .15 \\
\hline & BERT-ti & 67.62 & 47.71 & 58.46 & 47.89 & 63.86 & 55.04 & 56.76 \\
\hline & BERT-small & 61.90 & 44.50 & 56.41 & 47.89 & 71.08 & 51.77 & 55.59 \\
\hline & BERT-base & 64.76 & 47.25 & 49.74 & 58.42 & 74.10 & 54.50 & 58.13 \\
\hline \multirow{10}{*}{$\mathbf{F}$} & CNN & 49.94 & 44.98 & 52.31 & 49.69 & 58.33 & 54.50 & 51.63 \\
\hline & BiLSTM & 43.94 & 44.47 & 48.32 & 47.85 & 56.49 & 51.77 & 48.81 \\
\hline & + Attention & 39.18 & 45.07 & 49.07 & 44.70 & 52.86 & 54.55 & 47.57 \\
\hline & RCNN & 51.01 & 47.41 & 50.39 & 49.82 & 58.30 & 57.01 & 52.32 \\
\hline & FastText & 50.06 & 45.11 & 48.98 & 48.21 & 57.95 & 53.90 & 50.70 \\
\hline & DPCNN & 49.74 & 44.59 & 48.76 & 47.79 & 57.71 & 53.92 & 50.42 \\
\hline & Transformer & 50.13 & 45.91 & 51.33 & 50.95 & 58.98 & 54.20 & 51.92 \\
\hline & BERT-ti & 50.84 & 41.94 & 49.14 & 44.07 & 53.00 & 53.16 & 69 \\
\hline & BERT-small & 47.91 & 41.95 & 48.78 & 48.15 & 53.76 & 53.98 & 49.09 \\
\hline & BERT-base & 51.32 & 45.78 & 45.97 & 53.11 & 58.29 & 55.87 & 51.72 \\
\hline \multirow{10}{*}{ A } & CNN & 66.17 & 72.37 & 64.37 & 61.72 & 62.89 & 62.72 & 65.04 \\
\hline & BiLSTM & 63.83 & 74.18 & 62.50 & 56.41 & 62.70 & 62.63 & 63.71 \\
\hline & + Attentio & 69.22 & 70.50 & 68.68 & 62.50 & 66.21 & 62.16 & 66.55 \\
\hline & RCNN & 70.61 & 74.94 & 54.74 & 56.87 & 63.67 & 55.91 & 62.79 \\
\hline & FastText & 66.00 & 70.30 & 56.90 & 59.38 & 62.30 & 60.83 & 62.62 \\
\hline & DPCNN & 66.26 & 74.18 & 55.60 & 53.91 & 62.50 & 62.16 & 62.44 \\
\hline & Transforı & 66.09 & 67.68 & 55.32 & 63.59 & 63.87 & 61.78 & 63.06 \\
\hline & BERT-tiny & 64.17 & 70.95 & 66.09 & 66.28 & 63.57 & 66.32 & 66.23 \\
\hline & BERT & 63.13 & 72.92 & 66.81 & 71.39 & 60.66 & 69.35 & 67.38 \\
\hline & BERT-base & 66.35 & 75.39 & 67.24 & 71.39 & 65.89 & 70.10 & 69.39 \\
\hline
\end{tabular}

Table 16: Performance of text classification in predicting the scripts performed by specific leading comedians (0: Xiaobao SONG, 1: Yuepeng YUE, 2: Ling JIA, 3: Xiaofei ZHANG, 4: Teng SHEN, 5: Bing JIA).

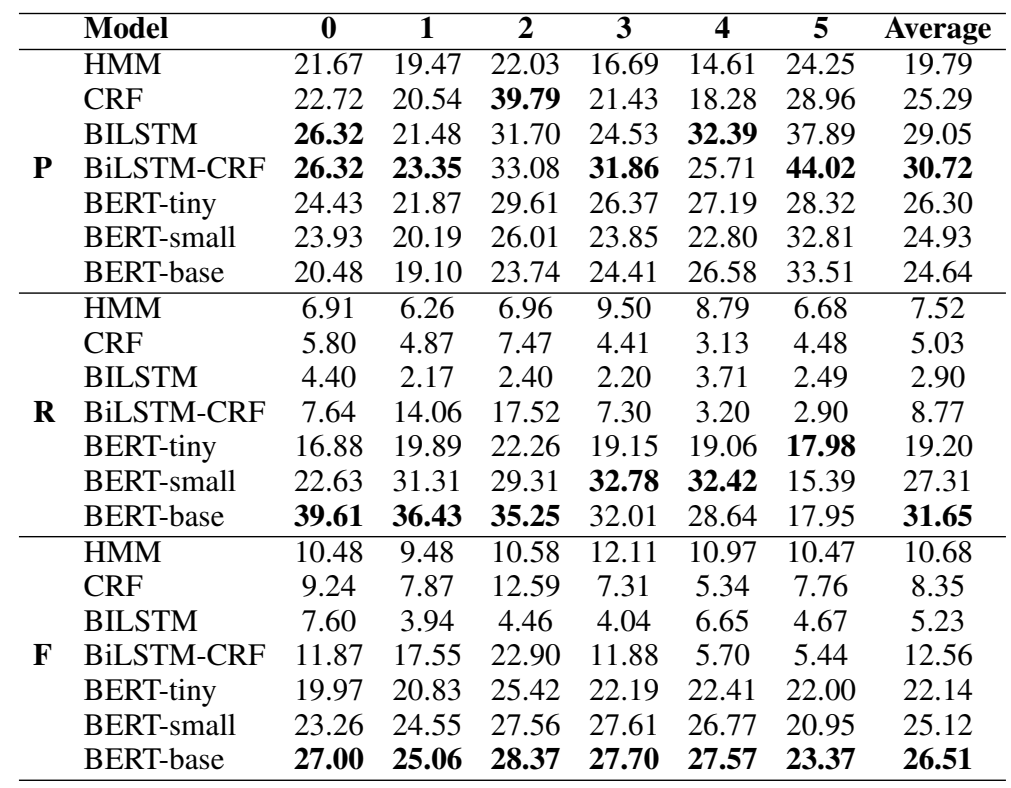

Table 17: Performance of information extraction in predicting the scripts performed by specific leading comedians. 


\begin{tabular}{|c|c|c|c|c|}
\hline $\begin{array}{c}\text { Performance } \\
\text { Title }\end{array}$ & Translation & Comedians & Source & Translation \\
\hline 扶不扶 & Help Her Up or Not & eng SHEN etc. & 央视春晚 & CCTV Chinese New Year Gala \\
\hline 碰瓷 & An Incident-Faking Extortionist & Xiaobao SONG etc. & 欢乐喜剧人(第一季) & Joyful Comedians (Season 1) \\
\hline 一念天堂 & Heaven or Hell? & Teng SHEN etc. & 欢乐喜剧人(第一季) & Joyful Comedians (Season 1) \\
\hline 纯闺蜜 & We Are Pure Besties & ng SHEN etc. & 欢乐喜剧人(第一季) & Joyful Comedians (Season 1) \\
\hline 以貌取人 & Judge By Appearances & Xiaobao SONG etc. & 辽宁卫视春晚 & LNTV Chinese New Year Gala \\
\hline 闺蜜小时代之怀孕 & Story of My Bestie - Pregnancy & Xiaofei ZHANG etc. & 喜剧班的春天(第一季) & Comedy Class of Spring (Season 1) \\
\hline 非一般的爱情 & Unusual Love & Yunpeng YUE and Yue Sun & 欢乐喜剧人(第二季) & Joyful Comedians (Season 2) \\
\hline 选妃记 & Select Imperial Concubine & Xiaobao SONG etc. & 王牌对王牌(第一季) & Ace VS Ace (Season 1) \\
\hline 暴走街区 & Violent Teenagers & aofei ZHANG etc. & 欢乐喜剧人(第三季) & Joyful Comedians (Season 3) \\
\hline 前男友前女友 & Ex-boyfri & 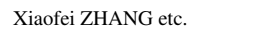 & E(第二季) & Comedy Class of Spring (Season 2) \\
\hline 落跑姐妹之还债 & Escap & ing IIA & 喜剧班的春天(第二季) & Comedy Class of Sp \\
\hline 公交故事之让座 & Give Up S & c. & 喜剧班的春天(第二季) & Comedy Class of Spring (Season 2) \\
\hline 贾总的演讲 & Manager JIA & ng JIA etc. & 笑声传奇(第一季) & Lengend of Laughter (Season 1) \\
\hline 一碗元宵 & A Bowl of Yuanxiao & iaofei ZHANG etc. & 湖南卫视元宵喜乐会 & Mango TV Lantern Festival Party \\
\hline 非诚来扰 & th Me If You Are Not Sincere & NG etc. & 视春晚 & LNTV Chinese New Year Gala \\
\hline 学车 & Learn & & 央视 & CCTV Chin \\
\hline 爱回家 & Love Back Home & etc. & 东方工 & Tomato TV Chinese New Year Gala \\
\hline 幸福牛家村 & Happy Niu Families' Village & Xiaofei ZHANG etc. & 江苏卫视春晚 & JSTV Chinese New Year Gala \\
\hline 关于爱情 & Something About Love & Yunpeng YUE and Yue Sun & 辽宁卫视春晚 & LNTV Chinese New Year Gala \\
\hline 懒汉相亲 & Idler's Blind Date & Ling JIA etc. & 王牌对王牌(第4季) & Ace VS Ace (Season 4) \\
\hline 啼笑皆非 & Not Know Whether to Laugh or Cry & Ling JIA etc. & 央视春晚 & CCTV Chinese New Year Gala \\
\hline & Grab The Best Seat In The Classroom for My Children & Teng SHEN etc. & 央视春晚 & CCTV Chinese New Year Gala \\
\hline 了 & Arrogant You & Yunpeng YUE and Yue Sun & 贝春晚 & Tomato TV Chinese New Year Gala \\
\hline 乌龙快递 & Express Delivery for COVID-19 Frontline Fighters & Bing JIA & 欢乐喜剧人(第六季) & Joyful Comedians (Season 6) \\
\hline 父与子 & Father and Son & Bing JIA & 辽宁卫视春晚 & LNTV Chinese New Year Gala \\
\hline 猜谜语 & Guess Riddles & Yunpeng YUE and Yue Sun & 辽宁卫视春晚 & LNTV Chinese New Year Gala \\
\hline 想说爱你不容易 & Not Easy to Say Love You & Xiaobao SONG etc. & 山东卫视春晚 & SDTV-1 Chinese New Year Gala \\
\hline 生活趣谈 & Funny Stories in Life & Yunpeng YUE and Yue Sun & 央视春晚 & CCTV Chinese New Year Gala \\
\hline & Husband's Mother & Ling JIA etc. & 央视春晚 & CCTV Chinese New Year Gala \\
\hline 走讨场 & Go Through The Mo & Teng SHEN etc. & 央视春晚 & CCTV Chinese New Year Gala \\
\hline
\end{tabular}

Table 18: Full list of the selected comedy performances with their titles, leading comedians and source.

\begin{tabular}{|c|c|c|c|c|c|c|c|}
\hline $\begin{array}{c}\text { Performance } \\
\text { Title }\end{array}$ & Translation & Year & $\begin{array}{c}\text { Duration } \\
\text { (mins) }\end{array}$ & Number of Lines & $\begin{array}{c}\text { Laughter Rate } \\
\text { (Line-Level) }\end{array}$ & Number of Characters & $\begin{array}{c}\text { Laugher Rate } \\
\text { (Character-Level) }\end{array}$ \\
\hline 扶不扶 & Help Her Up or Not & 2014 & 14.75 & 114 & $44.74 \%$ & 3411 & $10.29 \%$ \\
\hline 碰瓷 & An Incident-Faking Extortionist & 2015 & 16.07 & 202 & $23.27 \%$ & 3902 & $8.69 \%$ \\
\hline 一念天堂 & Heaven or Hell? & 2015 & 10.92 & 55 & $32.73 \%$ & 1518 & $9.55 \%$ \\
\hline 纯闺蜜 & We Are Pure Besties & 2015 & 11.3 & 85 & $35.29 \%$ & 2468 & $7.86 \%$ \\
\hline 以貌取人 & Judge By Appearances & 2015 & 12.87 & 220 & $31.36 \%$ & 3919 & $9.72 \%$ \\
\hline 闺蜜小时代之怀孕 & Story of My Bestie - Pregnancy & 2015 & 8.5 & 81 & $33.33 \%$ & 1894 & $7.44 \%$ \\
\hline 非一般的爱情 & Unusual Love & 2016 & 41.68 & 317 & $27.76 \%$ & 4836 & $10.36 \%$ \\
\hline 选妃记 & Select Imperial Concubine & 2016 & 12.62 & 141 & $21.28 \%$ & 2522 & $9.79 \%$ \\
\hline 暴走街区 & Violent Teenagers & 2017 & 12.67 & 190 & $20 \%$ & 4768 & $4.8 \%$ \\
\hline 前男友前女友 & Ex-boyfriend and Ex-girlfriend & 2017 & 10.43 & 106 & $19.81 \%$ & 2723 & $3.49 \%$ \\
\hline 落跑姐妹之还债 & Escapted Sisters - Repay a Debt & 2017 & 12.27 & 135 & $25.19 \%$ & 2894 & $6.91 \%$ \\
\hline 公交故事之让座 & Give Up Seats on a Bus & 2017 & 9.28 & 95 & $42.11 \%$ & 1795 & $10.53 \%$ \\
\hline 贾总的演讲 & Manager JIA's Presentation & 2017 & 23.5 & 228 & $36.4 \%$ & 5319 & $9.64 \%$ \\
\hline 一碗元宵 & A Bowl of Yuanxiao & 2018 & 17.58 & 177 & $31.07 \%$ & 4465 & $7.57 \%$ \\
\hline 非诚来扰 & Blind Date with Me If You Are Not Sincere & 2018 & 31.3 & 357 & $35.29 \%$ & 7854 & $10.06 \%$ \\
\hline 学车 & Learn Driving & 2018 & 14.83 & 165 & $30.3 \%$ & 3306 & $9.5 \%$ \\
\hline 爱回家 & Love Back Home & 2019 & 15.31 & 152 & $45.39 \%$ & 3738 & $12.79 \%$ \\
\hline 幸福牛家村 & Happy Niu Families' Village & 2019 & 12.18 & 131 & $31.7 \%$ & 3152 & $8.03 \%$ \\
\hline 关于爱情 & Something About Love & 2019 & 21.83 & 590 & $21.53 \%$ & 8044 & $7.91 \%$ \\
\hline 懒汉相亲 & Idler's Blind Date & 2019 & 8.77 & 101 & $18.81 \%$ & 1946 & $5.4 \%$ \\
\hline 啼笑皆非 & Not Know Whether to Laugh or Cry & 2019 & 17.72 & 178 & $24.72 \%$ & 4726 & $5.99 \%$ \\
\hline 占位子 & Grab The Best Seat In The Classroom for My Children & 2019 & 14 & 140 & $29.29 \%$ & 3786 & $6.15 \%$ \\
\hline 你膨胀了 & Arrogant You & 2020 & 12.65 & 241 & $29.88 \%$ & 3797 & $13.12 \%$ \\
\hline 乌龙快递 & Express Delivery for COVID-19 Frontline Fighters & 2020 & 15.43 & 195 & $24.1 \%$ & 4672 & $7.3 \%$ \\
\hline 父与子 & Father and Son & 2020 & 23.33 & 317 & $37.22 \%$ & 6343 & $10.2 \%$ \\
\hline 猜谜语 & Guess Riddles & 2020 & 19.4 & 523 & $18.93 \%$ & 7142 & $7.24 \%$ \\
\hline 想说爱你不容易 & Not Easy to Say Love You & 2020 & 12.53 & 230 & $18.7 \%$ & 4170 & $5.85 \%$ \\
\hline 生活趣谈 & Funny Stories in Life & 2020 & 11.5 & 312 & $16.03 \%$ & 4122 & $5.09 \%$ \\
\hline 婆婆妈妈 & Husband's Mother & 2020 & 16.42 & 187 & $31.02 \%$ & 4188 & $8.55 \%$ \\
\hline 走过场 & Go Through The Motions & 2020 & 11.8 & 122 & $21.31 \%$ & 3031 & $4.88 \%$ \\
\hline
\end{tabular}

Table 19: Full list of the selected comedy performances with their titles, years, duration, number of lines/characters and laughter rate at line/character-level. 